\title{
Small Molecule Inhibitors to Disrupt Protein-protein Interactions of Heat Shock Protein 90 Chaperone Machinery
}

\author{
Young Ho Seo \\ College of Pharmacy, Keimyung University, Daegu, Korea
}

\begin{abstract}
Heat shock protein 90 (Hsp90) is an adenosine triphosphate dependent molecular chaperone in eukaryotic cells that regulates the activation and maintenance of numerous regulatory and signaling proteins including epidermal growth factor receptor, human epidermal growth factor receptor 2, mesenchymal-epithelial transition factor, cyclin-dependent kinase-4, protein kinase B, hypoxia-inducible factor $1 \alpha$, and matrix metalloproteinase-2. Since many of Hsp90 clients are oncogenic proteins, Hsp90 has become an attractive therapeutic target for treatment of cancer. To discover small molecule inhibitors targeting Hsp90 chaperone machinery, several strategies have been employed, which results in three classes of inhibitors such as N-terminal inhibitors, C-terminal inhibitors, and inhibitors disrupting protein-protein interactions of Hsp90 chaperone machinery. Developing small molecule inhibitors that modulate protein-protein interactions of Hsp90 is a challenging task, although it offers many alternative opportunities for therapeutic intervention. The lack of well-defined binding pocket and starting points for drug design challenges medicinal chemists to discover small molecule inhibitors disrupting protein-protein interactions of Hsp90. The present review will focus on the current studies on small molecule inhibitors disrupting protein-protein interactions of Hsp90 chaperone machinery, provide biological background on the structure, function and mechanism of Hsp90's protein-protein interactions, and discuss the challenges and promise of its small molecule modulations.
\end{abstract}

(J Cancer Prev 2015;20:5-11)

Key Words: Heat shock protein 90, Protein-protein interaction, Small molecule, Antagonists \& Inhibitors

\section{INTRODUCTION}

Over the last two decades, several cancer drugs that target a single genetic abnormality have been discovered as so called 'targeted cancer drugs' and eradicated cancer cells in more specific ways without undesired side effects. ${ }^{1}$ Despite their superiority in the therapeutic efficacy and selectivity, these drugs often encounter the emergence of drug-resistance in cancer chemotherapy and that remains a major obstacle to cure cancers. Most cancers are heterogeneous and driven by multiple genetic abnormalities and hence, it is being recognized that cancers are more likely invincible by a single-targeted drug. ${ }^{2,3}$ In this regard, Heat shock protein 90 (Hsp90) provides a number of advantages to overcome drug resistance and has become an attractive therapeutic target for treatment of cancer. Hsp90 is an essential molecular chaperone in eukaryotic cells with an important role in activation and maintenance of numerous regulatory and signaling proteins including epidermal growth factor receptor (EGFR), human epidermal growth factor receptor 2 (Her2), mesenchymalepithelial transition factor (Met), cyclin-dependent kinase-4 (Cdk4), protein kinase B (Akt/PKB), hypoxia-inducible factor $1 \alpha$ (HIF-1 $\alpha$ ), and matrix metalloproteinase-2 (MMP2). ${ }^{4}$ Disruption of Hsp90 folding machinery induces client proteins degradation via the ubiquitin-proteasome pathway, which leads to cell death. Hsp90 is constitutively expressed at 2 to 10 fold higher levels in cancer cells than their normal counterparts. ${ }^{5,6}$ Hsp90 requires a series of cochaperones to form a complex for its function and cell division cycle 37 (Cdc37), Heat shock protein 70 (Hsp70), Heat

Received February 10, 2015, Revised February 23, 2015, Accepted February 23, 2015

Correspondence to: Young Ho Seo

College of Pharmacy, Keimyung University, 1095 Dalgubeol-daero, Dalseo-gu, Daegu 704-919, Korea

Tel: +82-53-580-6639, Fax: +82-53-580-5164, E-mail: seoyho@kmu.ac.kr, ORCID: Young Ho Seo, http://orcid.org/0000-0002-2268-8761

Copyright (C) 2015 Korean Society of Cancer Prevention

(c) This is an Open Access article distributed under the terms of the Creative Commons Attribution Non-Commercial License (http://creativecommons. org/icenses/by-nc/3.0) which permits unrestricted non-commercial use, distribution, and reproduction in any medium, provided the original work is properly cited. 


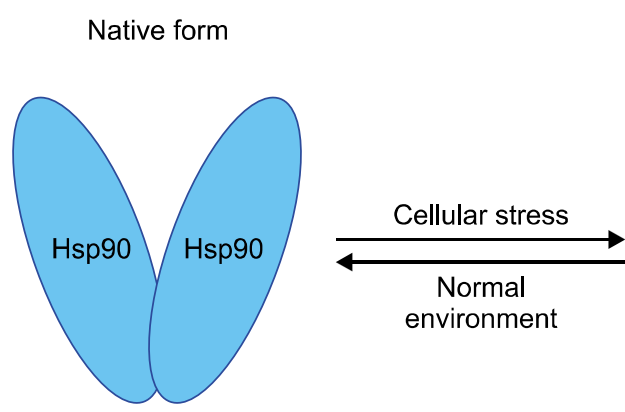

Normal cells

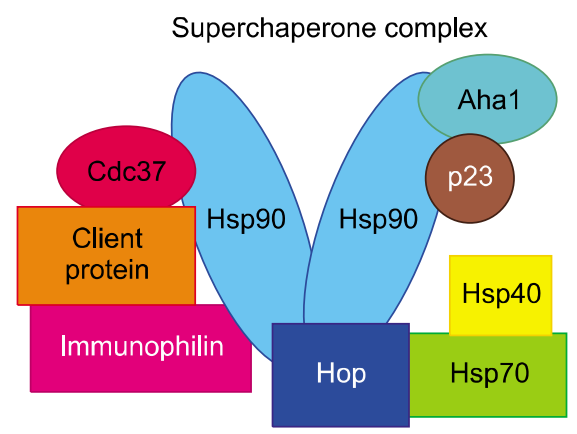

Cancer cells
Figure 1. Superchaperone complex of heat shock protein 90 (Hsp90) in cancer cells compared to normal cells. Cdc37, cell division cycle 37; Hop, Hsp organizing protein. shock protein 40 (Hsp40), Hsp organizing protein (Hop), p23, activator of Hsp90 ATPase (Aha1), and immunophilins belong to the cochaperones (Fig. 1).,8 In cancer cells, the cochaperones assemble into a superchaperone complex with Hsp90 and they assist Hsp90 to carry out its chaperoning job. By contrast, Hsp90 from normal cells does not generally form a superchaperone complex and has lower ATPase activity compared to cancer cells. ${ }^{9,10}$ Besides, it is reported that the binding affinity of Hsp90 inhibitor 17-AAG to Hsp90 in cancer cells is approximately 100 times stronger than in normal cells. ${ }^{9,11}$ Therefore, Hsp90 present in cancer cells forms a superchaperone complex and displays higher susceptibility to the inhibition by Hsp90 inhibitors than normal cells. Considering that therapeutic selectivity for cancer cells over normal cells is the Holy Grail in the war on cancer, the differential property of Hsp90 in cancer cells over normal cells shows particular promise in cancer chemotherapy.

Three major strategies have been employed to discover Hsp90 inhibitors. Most widely used strategy in academia and the pharmaceutical industry is to target ATP-binding pocket of Hsp90's N-terminal domain. ${ }^{12-18}$ To date, hundreds of N-terminal inhibitors have been discovered. Inhibitors to target adenosine triphosphate (ATP)-binding pocket of $\mathrm{N}$-terminal domain are considered to be the most effective as an anticancer agent. Currently, there are thirteen Hsp90 inhibitors undergoing clinical developments and all Hsp90 inhibitors under clinical evaluation are classified into N-terminal inhibitors. ${ }^{19}$ Despite the significant progress of this approach, N-terminal inhibitors induce the pro-survival heat shock response, which is speculated to limit their utility as anticancer therapy. ${ }^{4,20}$ Another strategy employed to inhibit Hsp90 function is to block the C-terminal nucleotidebinding domain that exhibits allosteric control over both substrates and ATP-binding site of N-terminal domain. ${ }^{21-26}$ Unlike $\mathrm{N}$-terminal inhibitors, $\mathrm{C}$-terminal inhibitors have not advanced to clinical investigations probably due to the absence of the co-crystal structure with their inhibitors. Intriguingly, C-terminal inhibitors are reported not to induce the heat shock response that is believed to be a detrimental consequence of $\mathrm{N}$-terminal inhibitors. ${ }^{27}$ Lastly, the strategy to disrupt protein-protein interactions of Hsp90 has been investigated as an alternative to modulate Hsp90 chaperone machinery. A small number of studies on the inhibition of protein-protein interactions have been conducted up to date. ${ }^{28-32}$ The present review will focus on the recent developments of the inhibitors disrupting protein-protein interactions of Hsp90 chaperone machinery and provide an overview on their biological insights and therapeutic implications.

\section{MAIN SUBJECT}

1. Inhibitors to disrupt heat shock protein 90-heat shock protein organizing protein interaction

Hsp organizing protein (Hop) is an abundant, highly conserved eukaryotic protein that mediates the association of the molecular chaperones, Hsp90 and Hsp70,33.34 The current consensus model for Hsp90 mediated protein folding assumes that a newly synthesized polypeptide is first chaperoned by Hsp70 and then transferred to Hsp90 for the correct folding and maturation (Fig. 2). To do so, Hsp70 and Hsp90 are brought into close proximity, via their interactions with Hop. C-terminal peptides of Hsp70 and Hsp90 independently interact with two tetratricopeptide repeat (TPR) domains of Hop, TPR1 and TPR2A respectively, and form a multichaperone complex of Hsp70-Hop-Hsp90. ${ }^{33,34}$ If Hsp90 is blocked to interact with TPR2A domain of Hop, a multichaperone complex of Hsp70-Hop-Hsp90 can no longer form. Besides, mounting evidence has demonstrated that the interaction of Hsp90 with Hop via the TPR2A is critically essential for Hsp90 chaperoning function. Therefore, the blockage of Hsp70-HopHsp90 folding relay system will lead to the ubiquitination and proteasomal degradation of Hsp90 client proteins.

To discover inhibitors disrupting Hsp90-Hop interactions, Yi and Regan ${ }^{31}$ performed an AlphaScreen technology based 


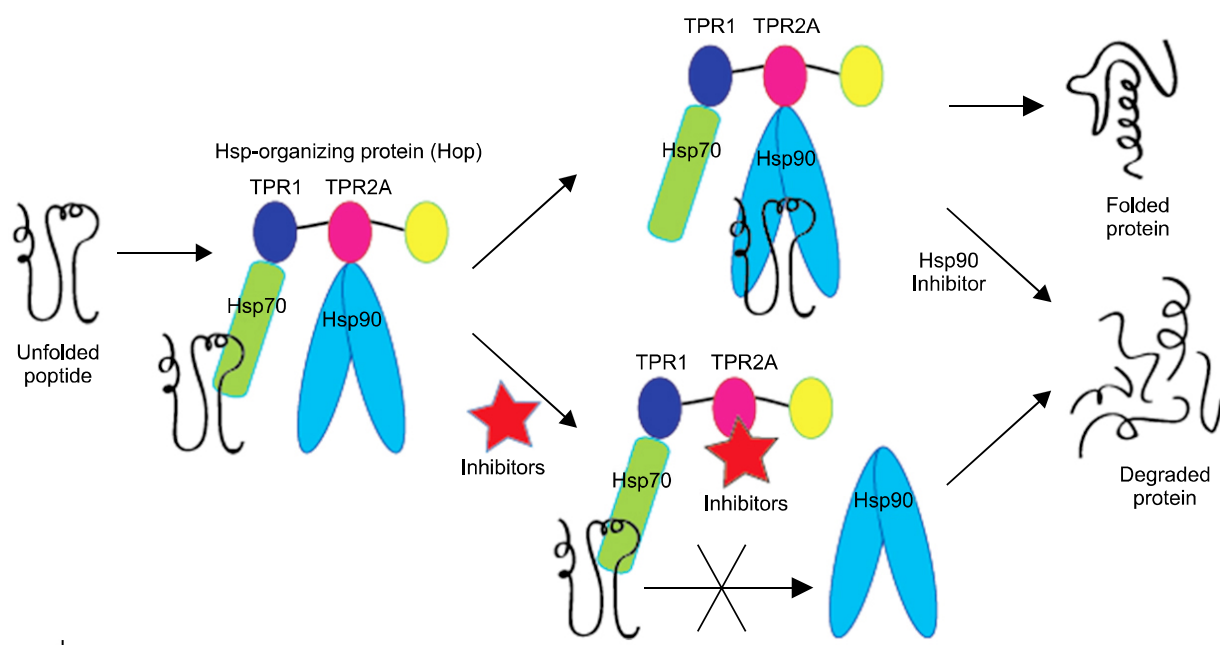<smiles>CCCc1nc2c(=O)n([N+](=O)[O-])c(=O)nc-2n(N)n1</smiles><smiles>CCn1nc(-c2ccccc2)nc2c(=O)n(C)c(=O)nc1-2</smiles>

$3.60 \mathrm{e}-7 \mathrm{M}$<smiles>Cn1nc(-c2ccccc2)nc2c(=O)n(C)c(=O)nc1-2</smiles>

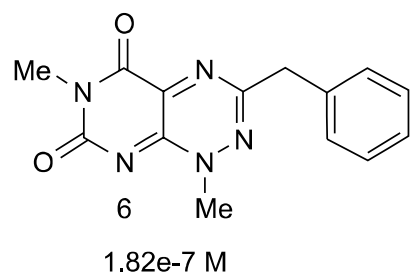

Figure 2. Schematic illustration of Hsp90-Hop-Hsp70 folding relay system. TPR, tetratricopeptide repeat; Hsp, heat shock protein.<smiles>Cn1nc(-c2cccs2)nc2c(=O)n(C)c(=O)nc1-2</smiles>

3.65e-7 M high-throughput in vitro screen to identify six compounds that disrupted the interaction of Hsp90-Hop (Fig. 3). ${ }^{29}$ In the AlphaScreen technology, the excitation of the donor bead bound to Hsp90 at $680 \mathrm{~nm}$ produces singlet state oxygen species, which then diffuse up to $200 \mathrm{~nm}$ and activate flurophores in the acceptor bead bound to Hop. As a result, protein-protein interaction of Hsp90 with Hop emits light at 520 to $620 \mathrm{~nm}$. To rule out any false positives in the AlphaScreen technology, they executed fluorescence polarization assay and isothermal titration calorimetry (ITC) and confirmed the six compounds. ITC study showed that compound 1 directly bound to TPR2A domain of Hop with dissociation constant $\left(\mathrm{K}_{\mathrm{d}}\right)$ value of $16 \mu \mathrm{M}$ and a $1: 1$ binding stoichiometry. In addition, they demonstrated that compound 1 inhibited Her2-positive human breast cancer cell lines BT474. Interestingly, these compounds did not induce the upregulation of Hsp70, which was speculated to limit the efficacy of Hsp90 inhibitors under certain circumstances. ${ }^{35}$ All six compounds that they identified have a 7-azapteridine ring system in common and displayed similar activities, with no substitution effect causing a dramatic change in activity. They speculated that $\mathrm{C} 3$ position of 1,2,4-triazine ring that they modified substitution was probably not a key position and that may exert a relatively flat structure-activity relationship (SAR). Up to date, the optimization and SAR studies of the hit compounds have not been reported. Accordingly, further extensive SAR studies along with structural characterization of TPR2A-small molecule complex will provide more detailed information about TPR2A-small molecule interactions and direct how the compounds can be chemically modified for the higher potency and specificity. Inhibitors disrupting Hsp90-Hop interaction could eventually lead to efficacious alternatives to Hsp90 N-terminal inhibitors.

\section{Inhibitors to disrupt heat shock protein 90-cell division cycle 37 interaction}

Cdc37 was originally identified in mammalian cells as a component of a protein complex involving Hsp90 and the Rous 
sarcoma virus protein, pp $60^{\text {v-src } 36}$ The cochaperone adaptor Cdc37, also known as p50, pp50, and p50 $0^{\text {cdc37 }}$ plays an important role in regulating the Hsp90 chaperone cycle by assisting the recruitment of client proteins that include numerous oncogenic kinases. ${ }^{37-40}$ Accumulating evidence suggested that the overexpression of $\mathrm{Cdc} 37$ is related to the oncogenic transformation and Cdc37 is required for the folding and the maturation of Hsp90-dependent protein kinases including Her2, Raf-1, Cdk4, and Akt. Consequently, Hsp90-Cdc37 chaperone complex maintains a population of protein kinases in cells, which is implicated in signal transduction, cell proliferation, and survival. The critical role of the Hsp90-Cdc37 chaperone complex makes it an attracting therapeutic target for anti-cancer drug development.

The co-crystal structure of Hsp90 with Cdc37 revealed that the

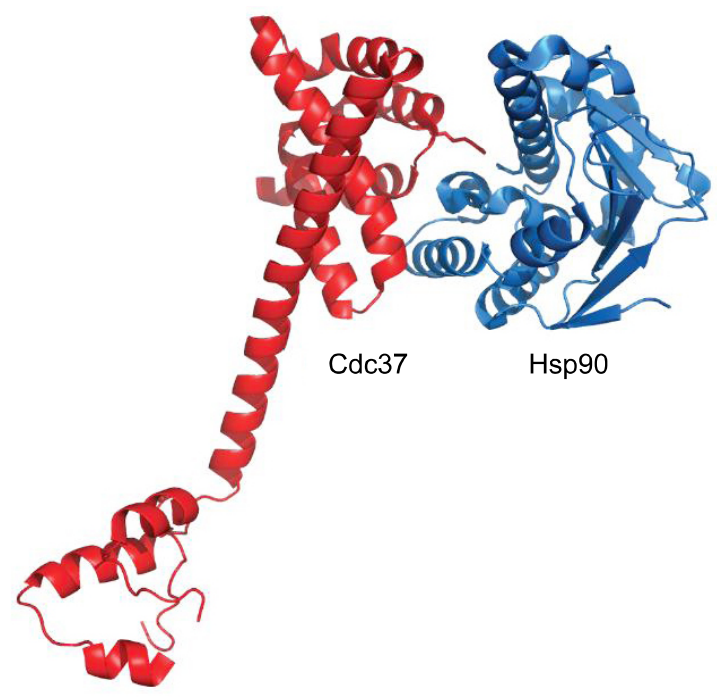

Figure 4. X-ray crystal structure of Hsp90-Cdc37 complex (PDB code: 1US7) showing the N-terminal domain of Hsp90 (blue) and the C-terminal domain of $\mathrm{Cdc} 37$ (red). The figure was generated using PyMol (DeLano Scientific). Hsp90, heat shock protein 90; Cdc37, cell division cycle 37. large helical domain in the C-terminal of Cdc37 binds to the $\mathrm{N}$-terminal domain of Hsp90, which is believed to close over bound ATP in ATP-binding pocket of Hsp90's N-terminal domain (Fig. 4). ${ }^{37}$ The core interactions of Hsp90-Cdc37 complex involves a relatively flat hydrophobic patch formed by the hydrophobic residues of Hsp90 and Cdc37. Despite the interacting regions of Hsp90-Cdc37 are located in close proximity to the ATP-binding pocket of Hsp90's N-terminal domain, most N-terminal inhibitors do not disrupt Hsp90-Cdc37 interactions. ${ }^{37}$

In 2008, Zhang et al. ${ }^{32}$ first identified that a natural product, celastrol blocked Cdc37 binding to Hsp90 (Fig. 5). Celastrol is a quinone methide triterpene isolated from Tripterygium wilfordii Hook F (the Chinese Thunder of God vine). ${ }^{41}$ Using ATP-sepharose binding and co-immunoprecipitation assay, they concluded that celastrol disrupted Hsp90-Cdc37 without blocking ATP binding to Hsp90. The molecular modeling predicted that celastrol binds to the mouth region of ATP-binding pocket in N-terminal domain of Hsp90 and induced a major conformational change in the binding pocket, resulting in a significant decrease of the binding affinity of Cdc37 to Hsp90. The study demonstrated that celastrol disrupted only Hsp90-Cdc37 complex without affecting other Hsp90-cochaperone interactions such as Hsp90-Hop and Hsp90p23. As a consequence, celastrol degraded Hsp90 client proteins including Akt and Cdk4 in pancreatic cancer cells. In Panc-1 cell xenograft mice, celastrol inhibited $80 \%$ of tumor growth, effect of which is slightly better than N-terminal inhibitor, geldanamycine. Interestingly, they observed that celastrol induced heat shock response, leading to the upregulation of Hsp70. The finding may explain that celastrol has multiple mechanisms of action together with Hsp90-Cdc37 disruption.

In 2012, the same research group reported that a natural product, sulforaphane disrupted Hsp90-Cdc37 complex to impair the growth of pancreatic cancer. ${ }^{28}$ Sulforaphane is a major compound from broccoli and broccoli sprout, containing an

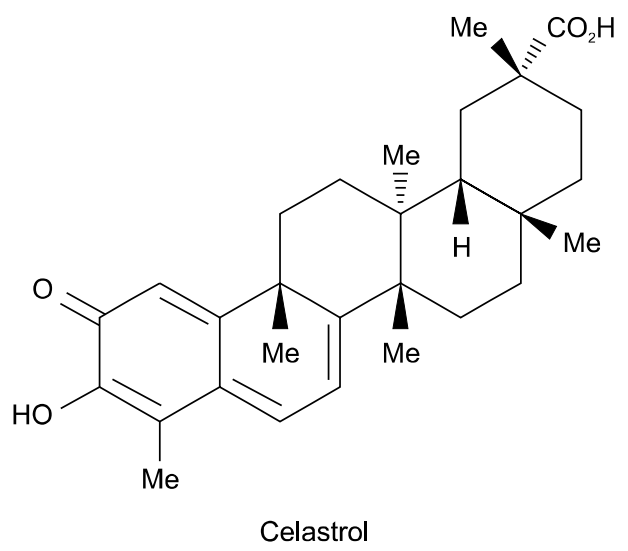<smiles>CS(=O)CCCCN=C=S</smiles>

Sulforaphane
Figure 5. Structures of celastrol and sulforaphane. 
isothiocyanate structure on one end (Fig. 5). ${ }^{42,43}$ ATP-sepharose binding assay displayed that sulforaphane inhibited Hsp90 function through an ATP-binding independent manner. Hsp90 co-immunoprecipitation, proteolytic fingerprinting assay, nuclear magnetic resonance spectroscopy, and LC-MS peptide mapping revealed that sulforaphane modified N-terminal domain of Hsp90 and disrupted Hsp90-Cdc37 complex. In this study, sulforaphane promoted the proteasomal degradation of Hsp90 client proteins such as Akt, Cdk4, and mutant p53, resulting in pancreatic cancer cell death.

\section{Inhibitors to disrupt heat shock protein 90-human epidermal growth factor receptor 2 interaction}

Few studies have been reported to discover small molecule inhibitors specifically inhibiting Hsp90-client interactions. It is probably due to the lack of information on how exactly a single client protein interacts with Hsp90. Nonetheless, Yan et al. ${ }^{30}$ reported that emodin azide methyl anthraquinone derivative (AMAD) blocked Her2 binding to Hsp90 (Fig. 6). Emodin AMAD is an emodin azide methyl anthraquione derivative, obtained from chemical modification of emodin. Emodin is a natural product extracted from nature's giant knotweed rhizome of traditional Chinese herbs. ${ }^{44}$ The study revealed that AMAD significantly impaired the binding of Her2 to Hsp90, promoted Her2 ubiquitination and destroyed the plasma membrane location of Her2. To investigate the molecular mechanism of emodin AMAD in blocking Hsp90-Her2 complex, they performed in silico docking study. The docking study demonstrated that emodin AMAD could bind to both Hsp90 and Her2 nucleotide binding sites. They speculated that emodin AMAD either binds to ATP-binding pocket of Hsp90 or Her2, resulting in structural distortion of chaperone complexes and subsequent degradation of Her2.

\section{CONCLUSION}

Hsp90 is responsible for diverse cellular function in normal cells, but also involved in malignant transformation and disease progression. Therefore, Hsp90 has become an active molecular target for the treatment of many diseases, including cancer, neurodegenerative diseases, and pathogenic infections. At present, clinical applications for Hsp90 modulation primarily focus on the treatment of cancer and the progress has been made using Hsp90-targeted therapies. Although N-terminal inhibitors, C-terminal inhibitors, and inhibitors disrupting protein-protein interactions of Hsp 90 have been investigated as anticancer

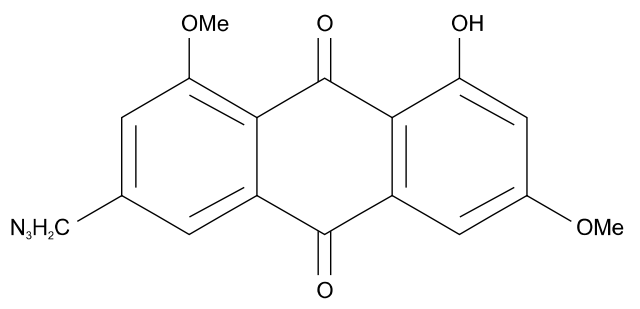

Figure 6. Structure of emodin azide methyl anthraquinone derivative.

agents, only competitive inhibitors that target N-terminal ATPbinding site have entered into clinical developments. Vast amount of studies were conducted to discover N-terminal inhibitors in clinical applications. To date, however, there is no Food and Drug Administration in USA approved Hsp90 inhibitor. Accordingly, alternative strategy might provide an opportunity for the development of Hsp90 inhibitors with clinical applications and inhibiting protein-protein interactions of Hsp90 chaperone machinery may represent a promising strategy to find clinical application in cancer research. In this review, we overviewed the current studies of small molecule inhibitors disrupting protein-protein interactions of Hsp90 chaperone machinery, their biological importance, and therapeutic applications for cancer treatment.

\section{ACKNOWLEDGMENTS}

This research was supported by Basic Science Research Program through the National Research Foundation of Korea (NRF) funded by the Ministry of Education, Science and Technology (2011-0023605).

\section{CONFLICTS OF INTEREST}

No potential conflicts of interest were disclosed.

\section{REFERENCES}

1. Aggarwal S. Targeted cancer therapies. Nat Rev Drug Discov 2010; 9:427-8.

2. Boran $A D$, Iyengar R. Systems approaches to polypharmacology and drug discovery. Curr Opin Drug Discov Devel 2010;13: 297-309.

3. Petrelli A, Giordano S. From single- to multi-target drugs in cancer therapy: when aspecificity becomes an advantage. Curr Med Chem 2008; 15:422-32.

4. Mahalingam D, Swords R, Carew JS, Nawrocki ST, Bhalla K, Giles FJ. Targeting HSP90 for cancer therapy. Br J Cancer 2009;100: 1523-9. 
5. Ferrarini M, Heltai S, Zocchi MR, Rugarli C. Unusual expression and localization of heat-shock proteins in human tumor cells. Int J Cancer 1992;51:613-9.

6. Whitesell L, Lindquist SL. HSP90 and the chaperoning of cancer. Nat Rev Cancer 2005;5:761-72.

7. Workman P. Altered states: selectively drugging the Hsp90 cancer chaperone. Trends Mol Med 2004;10:47-51.

8. Pratt WB, Dittmar KD. Studies with purified chaperones advance the understanding of the mechanism of glucocorticoid receptorhsp90 heterocomplex assembly. Trends Endocrinol Metab 1998; 9:244-52.

9. Kamal A, Thao L, Sensintaffar J, Zhang L, Boehm MF, Fritz LC, et al. A high-affinity conformation of Hsp90 confers tumour selectivity on Hsp90 inhibitors. Nature 2003:425:407-10.

10. Isaacs JS, Xu W, Neckers L. Heat shock protein 90 as a molecular target for cancer therapeutics. Cancer Cell 2003;3:213-7.

11. Kelland LR, Sharp SY, Rogers PM, Myers TG, Workman P. DTDiaphorase expression and tumor cell sensitivity to 17-allylamino, 17-demethoxygeldanamycin, an inhibitor of heat shock protein 90. J Natl Cancer Inst. 1999;91:1940-9.

12. Brough PA, Aherne W, Barril X, Borgognoni J, Boxall K, Cansfield JE, et al. 4,5-diarylisoxazole Hsp90 chaperone inhibitors: potential therapeutic agents for the treatment of cancer. J Med Chem 2008:51:196-218.

13. Wright L, Barril X, Dymock B, Sheridan L, Surgenor A, Beswick M, et al. Structure-activity relationships in purine-based inhibitor binding to HSP90 isoforms. Chem Biol 2004;11:775-85.

14. Whitesell L, Mimnaugh EG, De Costa B, Myers CE, Neckers LM. Inhibition of heat shock protein HSP90-pp60v-src heteroprotein complex formation by benzoquinone ansamycins: essential role for stress proteins in oncogenic transformation. Proc Natl Acad Sci U S A 1994:91:8324-8.

15. Lee T, Seo YH. Targeting the hydrophobic region of Hsp90's ATP binding pocket with novel 1,3,5-triazines. Bioorg Med Chem Lett. 2013:23:6427-31.

16. Seo YH. Discovery of licochalcone a as a natural product inhibitor of Hsp90 and its effect on gefitinib resistance in non-small cell lung cancer (NSCLC). Bull Korean Chem Soc 2013;34:1917-20.

17. Jeong $\mathrm{CH}$, Park HB, Jang WJ, Jung SH, Seo YH. Discovery of hybrid Hsp90 inhibitors and their anti-neoplastic effects against gefitinib-resistant non-small cell lung cancer (NSCLC). Bioorg Med Chem Lett 2014:24:224-7.

18. Seo YH, Park SY. Synthesis of flavokawain analogues and their anti-neoplastic effects on drug-resistant cancer cells through Hsp90 inhibition. Bull Korean Chem Soc 2014:35:1154-8.

19. Sidera K, Patsavoudi E. HSP90 inhibitors: current development and potential in cancer therapy. Recent Pat Anticancer Drug Discov 2014;9:1-20.

20. Powers MV, Workman P. Inhibitors of the heat shock response: biology and pharmacology. FEBS Lett 2007;581:3758-69.

21. Marcu MG, Chadli A, Bouhouche I, Catelli M, Neckers LM. The heat shock protein 90 antagonist novobiocin interacts with a previously unrecognized ATP-binding domain in the carboxyl terminus of the chaperone. J Biol Chem 2000;275:37181-6.

22. Marcu MG, Schulte TW, Neckers L. Novobiocin and related coumarins and depletion of heat shock protein 90-dependent signaling proteins. J Natl Cancer Inst 2000;92:242-8.

23. Prodromou C, Siligardi G, O'Brien R, Woolfson DN, Regan L, Panaretou B, et al. Regulation of Hsp90 ATPase activity by tetra- tricopeptide repeat (TPR)-domain co-chaperones. EMBO J 1999; 18:754-62.

24. Csermely P, Schnaider T, Soti C, Prohászka Z, Nardai G. The 90-kDa molecular chaperone family: structure, function, and clinical applications. A comprehensive review. Pharmacol Ther 1998;79:129-68.

25. Allan RK, Mok D, Ward BK, Ratajczak T. Modulation of chaperone function and cochaperone interaction by novobiocin in the C-terminal domain of Hsp90: evidence that coumarin antibiotics disrupt Hsp90 dimerization. J Biol Chem. 2006;281:7161-71.

26. Kusuma BR, Khandelwal A, Gu W, Brown D, Liu W, Vielhauer G, et al. Synthesis and biological evaluation of coumarin replacements of novobiocin as Hsp90 inhibitors. Bioorg Med Chem 2014:22:1441-9.

27. Xia Y, Rocchi P, Iovanna JL, Peng L. Targeting heat shock response pathways to treat pancreatic cancer. Drug Discov Today 2012;17: 35-43.

28. Li Y, Karagöz GE, Seo YH, Zhang T, Jiang Y, Yu Y, et al. Sulforaphane inhibits pancreatic cancer through disrupting Hsp90-p50 (Cdc37) complex and direct interactions with amino acids residues of Hsp90. J Nutr Biochem 2012;23:1617-26.

29. Pimienta G, Herbert KM, Regan L. A compound that inhibits the HOP-Hsp90 complex formation and has unique killing effects in breast cancer cell lines. Mol Pharm 2011;8:2252-61.

30. Yan YY, Zheng LS, Zhang X, Chen LK, Singh S, Wang F, et al. Blockade of Her2/neu binding to Hsp90 by emodin azide methyl anthraquinone derivative induces proteasomal degradation of Her2/neu. Mol Pharm 2011;8:1687-97.

31. Yi F, Regan L. A novel class of small molecule inhibitors of Hsp90. ACS Chem Biol 2008;3:645-54.

32. Zhang T, Hamza A, Cao X, Wang B, Yu S, Zhan CG, et al. A novel Hsp90 inhibitor to disrupt Hsp90/Cdc37 complex against pancreatic cancer cells. Mol Cancer Ther 2008;7:162-70.

33. Chen S, Smith DF. Hop as an adaptor in the heat shock protein 70 (Hsp70) and hsp90 chaperone machinery. J Biol Chem 1998;273: 35194-200.

34. Scheufler C, Brinker A, Bourenkov G, Pegoraro S, Moroder L, Bartunik H, et al. Structure of TPR domain-peptide complexes: critical elements in the assembly of the Hsp70-Hsp90 multichaperone machine. Cell 2000;101:199-210.

35. Drysdale MJ, Brough PA, Massey A, Jensen MR, Schoepfer J. Targeting Hsp90 for the treatment of cancer. Curr Opin Drug Discov Devel 2006;9:483-95.

36. Brugge JS. Interaction of the Rous sarcoma virus protein pp60src with the cellular proteins pp50 and pp90. Curr Top Microbiol Immunol 1986;123:1-22.

37. Roe SM, Ali MM, Meyer P, Vaughan CK, Panaretou B, Piper PW, et al. The Mechanism of Hsp90 regulation by the protein kinase-specific cochaperone p50(cdc37). Cell 2004;116:87-98.

38. Pearl LH. Hsp90 and Cdc37: a chaperone cancer conspiracy. Curr Opin Genet Dev 2005;15:55-61.

39. Smith JR, Clarke PA, de Billy E, Workman P. Silencing the cochaperone CDC37 destabilizes kinase clients and sensitizes cancer cells to HSP90 inhibitors. Oncogene 2009;28:157-69.

40. Smith JR, Workman P. Targeting CDC37: an alternative, kinase-directed strategy for disruption of oncogenic chaperoning. Cell Cycle 2009;8:362-72.

41. Westerheide SD, Bosman JD, Mbadugha BN, Kawahara TL, Matsumoto G, Kim S, et al. Celastrols as inducers of the heat shock 
response and cytoprotection. J Biol Chem 2004:279:56053-60.

42. Fahey JW, Zhang Y, Talalay P. Broccoli sprouts: an exceptionally rich source of inducers of enzymes that protect against chemical carcinogens. Proc Natl Acad Sci U S A 1997:94:10367-72.

43. Conaway CC, Wang CX, Pittman B, Yang YM, Schwartz JE, Tian D, et al. Phenethyl isothiocyanate and sulforaphane and their N-acetylcysteine conjugates inhibit malignant progression of lung ad- enomas induced by tobacco carcinogens in $\mathrm{A} / \mathrm{J}$ mice. Cancer Res 2005:65:8548-57.

44. Yan Y, Su X, Liang Y, Zhang J, Shi C, Lu Y, et al. Emodin azide methyl anthraquinone derivative triggers mitochondrial-dependent cell apoptosis involving in caspase-8-mediated Bid cleavage. Mol Cancer Ther 2008;7:1688-97. 